MS \#04148

\title{
Prostate-specific antigen, Gleason sum and clinical T stage for predicting the need for radionuclide bone scan for prostate cancer patients in Japan
}

OSAMU ISHIZUKA, TOMOAKI TANABE, TSUYOSHI NAKAYAMA, MASAKO KAWAKAMI, YOSHIAKI KINEBUCHI AND OSAMU NISHIZAWA

Department of Urology, Shinshu University School of Medicine, Matsumoto, Japan

Correspondence: Osamu Ishizuka M.D., Ph.D.

Department of Urology,

Shinshu University School of Medicine

3-1-1 Asahi, Matsumoto 390-8621, Japan

Tel.: +81 26337 2661; Fax: +81 263373082

e-mail: ishizuk@hsp.md.shinshu-u.ac.jp

Running title: PSA, Gleason sum and radionuclide bone scan 


\section{Abstract}

Aim: In the study, we evaluated the relationship between PSA level and bone metastasis, between Gleason sum and bone metastasis and between clinical T stage and bone metastasis in Japanese patients.

Patients and Methods: Between November 1998 and June 2004, we performed ultrasound-guided biopsies on 709 patients (mean age: 70.5 years, range: 39 90). Prostate cancer was detected in 339 patients (47.8\%), 297 (87.6\%) of whom underwent a radionuclide bone scan. In close collaboration with the orthopedists, bone CT scans, bone MRI and/or plain X-P were performed for cases which were difficult to diagnose as bone metastasis by means of radionuclide bone scans only.

Results: We could detect 61 (20.6\%) bone metastasis cases in 296 patients. A simple linear regression analysis between log [PSA ] and bone metastasis $(n=296)$ produce a significant relationship $(p<0.05)$. If we set the cut-off PSA value of $15 \mathrm{ng} / \mathrm{ml}$ for indication of bone scan, the possibility of bone metastasis was $10 \%$. However, from our experience, there was no bone metastasis in the patients whose Gleason sums were under 5 , and in the patients whose Gleason sum were 5 and over, and the PSA levels were under 15, there was no bone metastasis. The rate of bone metastasis increased with the increase of PSA level. In the dinical T1-2 stage cases, there were the significant higher PSA levels in the cases with bone metastasis. In the T1-2 patients whose PSA levels were under 16, there was no bone metastasis.

Conclusions: From the analysis of PSA, Gleason sum and clinical T stage, we may not have to do bone scan for the patients whose PSA level is under 15 $\mathrm{ng} / \mathrm{ml}$ or Gleason sum is under 5 .

\section{Key words}

prostate cancer, prostate-specific antigen, radionuclide bone scan 


\section{Introduction}

The American Urological Association Prostate Cancer Guidelines Panel [1] stated that bone scanning "may no longer be necessary" for newly diagnosed patients with PSA of $<10 \mathrm{ng} / \mathrm{ml}$ and no skeletal symptoms. The National Comprehensive Cancer network recommends a bone scan for patients with clinical stage T-1 and T-2 disease only if their PSA is over $10 \mathrm{ng} / \mathrm{ml}$ or their Gleason score is 8 or above, and for all patients with clinical stage T-3 or T-4 disease or with bone symptoms [2]. The Society of Surgical Oncology's surgical practice guidelines for prostate cancer recommend a bone scan for preoperative evaluation only if the PSA is over $8 \mathrm{ng} / \mathrm{ml}$ [3]. O'Dowd et al extracted data from 142 articles in the Medline database and concluded that routine bone scanning is necessary for newly diagnosed asymptomatic patients only when the PSA is $10 \mathrm{ng} / \mathrm{ml}$ or more [4].

However, compared with the United States, the incidence and age-adjusted mortality rates for prostate cancer in Asian countries can be up to 10 times lower [5]. In Asians immigrants in the United States, the prostate cancer rates tend to increase over time to approach levels seen in the native US population. It is thus not clear whether the established bone scanning indication guidelines, which are mainly based on data from Europe and United States, are also applicable to Asians.

In this study, we evaluated the relationship between PSA level, Gleason sum, clinical T stage and bone metastasis. The results may become one of the useful tools for predicting the probability of bone metastasis and determining the indication for bone nuclide scans for Asians, especially Japanese. 


\section{Patients and methods}

From November 1998 to June 2004, we performed four transition zone core biopsies, in addition to sextant systemic ultrasound-guided peripheral zone biopsies, on 709 patients (mean age: 70.5 years; range: 39 - 90). Prostate cancer was detected in 339 patients (47.8\%), 297 (87.6\%) of whom underwent radionuclide bone scan, regardless of their PSA levels, Gleason sum and clinical T stages. Characteristics of the patients are summarized in Table 1.

\section{Biopsy technique and PSA determination}

Transrectal ultrasound-guided biopsies were performed in the sagital plane with a 7.0-MHz sector scanner (LPGIQ $\alpha 200$; GE Yokogawa Medical Systems, Tokyo, Japan) fitted with a biopsy guide using an 18-gauge needle driven by a spring-loaded biopsy gun. Endorectal xylocaine gel was used for ultrasonography of the prostate gland and six systematic biopsies aimed the peripheral zone were obtained from the left and right sides of the apex, the middle and the base of the prostate. We also obtained four additional biopsies aimed at the transition zone from the left and right sides of the apex and base of the prostate [6]. Total PSA serum concentrations were determined beforehand with the EIA method (Dai-Nippon Co., Osaka, Japan).

\section{Radionuclide bone scan}

Tc-99m MDP Radionuclide bone scans were performed soon after the pathological diagnosis of the prostate cancers. In close collaboration with the orthopedists, bone CT scans, bone MRI and/or plain X-P were performed for cases which were difficult to diagnose as bone metastasis by means of radionuclide bone scans only. 


\section{Statistics}

The relationships between log [PSA] level and bone metastasis rate were assessed by means of a simple linear regression, and correlation coefficient of $p$ $<0.05$ were considered significant. One-way factorial ANOVA followed by Scheffe's F-test was used for comparisons of PSA level and Gleason sum between bone metastasis group and non-bone metastasis group in each clinical T stage. $\mathrm{p}<0.05$ was accepted as significant. 


\section{Results}

A total of 297 radionuclide bone scan were performed, but a final diagnosis of bone metastasis could not be reached in one case in spite of an additional bone CT scan and bone MRI as well as consultation with the orthopedists. We could therefore eventually evaluate the relationship between PSA level and bone metastasis in 296 patients, in 61 (20.6\%) of whom bone metastasis was detected by means of radionuclide bone scans. The relationship between Gleason sum and bone metastasis could be assessed in 238 patients.

The results of a simple linear regression between log [PSA] level and bone metastasis are shown in Table 2 and Figure 1. The regression slope, regression intercept and correlation coefficient were 33.3, -25.6 and 0.949, respectively. The set of values showed a significant relationship $(p<0.05)$. If we set the cut-off PSA value of $15 \mathrm{ng} / \mathrm{ml}$ for indication of bone scan, the possibility of bone metastasis was 10\% (Figure 1). If we set the cut-off PSA value of 10 $\mathrm{ng} / \mathrm{ml}$, the possibility of bone metastasis was 5\% (Figure 1).

There was no bone metastasis in the patients whose Gleason sums were under 5 . In the patients whose Gleason sum were 5 and over, and the PSA levels were under 15 , there was no bone metastasis, however, the rate of bone metastasis was increased with the increase of PSA level (Table 3).

In the patients whose clinical stages were T1-2, there were the significant higher PSA levels in the cases with bone metastasis. In the T1-2 patients whose PSA levels were under 16, there was no bone metastasis. The Gleason sum was higher in the T1-2 cases with bone metastasis, however the difference was not significant (Table 4). 


\section{Discussion}

Chybowski and coworkers looked at 521 randomly selected patients in a retrospective study [7]. These patients, ranging from 44 to 92 years of age with a mean age of 70 years, all had untreated prostate cancer. All patients were given a digital rectal examination to establish clinical stage and underwent prostate biopsy or transurethral resection of the prostate to establish tumor grade. In addition, serum acid phosphatase, PAP and PSA were measured, and a radionuclide bone scan was administered. The investigators then examined these factors for correlation with bone-scan results. Local clinical stage, tumor grade, acid phosphatase, PAP and PSA all correlated positively with the incidence of a positive bone scan, each with a coefficient of $\mathrm{p}<0.0001$. They found that PSA was the best for predicting the results of a radionuclide bone scan when receiver operating characteristic (ROC) curves were used. They concluded that radionuclide bone scans are unnecessary for the staging of previously untreated prostate cancer patients who have no skeletal symptoms and a serum PSA value less than or equal to $10 \mathrm{ng} / \mathrm{ml}$. Pantelides and colleagues also attempted to determine the level of serum PSA that would predict osseous metastasis [8]. Fifty histologically confirmed but untreated prostate cancer patients were carefully monitored with bone scans during a long-term follow-up. They noted that a serum PSA level higher than $58 \mathrm{ng} / \mathrm{ml}$ yielded a $79 \%$ positive predictive accuracy for detecting skeletal disease and proposed that untreated prostate cancer patients with such a PSA level should undergo radionuclide bone imaging. In some cases, however, we detected bone metastasis in patients with low-level PSA and high-level Gleason sum.

The National Comprehensive Cancer network guidelines include the pathological tumor grade (Gleason score) as an indication of radionuclide bone 
scan [2]. In our study reported here, we examined the relationship between PSA level, Gleason sum, clinical T stage and the incidence of bone metastasis and our results indicate that PSA level were closely related to bone metastasis rates, and that there was no bone metastasis in the patients whose Gleason sums were under 5 . In the patients whose Gleason sum were 5 and over, and the PSA levels were under 15 , there was no bone metastasis, however, the rate of bone metastasis was increased with the increase of PSA level. In the T1-2 patients whose PSA levels were under 16, there was no bone metastasis. The Gleason sum was higher in the T1-2 cases with bone metastasis, however the difference was not significant. There may be a significant difference by the increase of the patients.

Previously, Kosuda et al. reported the multicenter retrospective study about the PSA level and the need for bone scan in Japan [8]. From the analysis of 1294 patients, they suggested that baseline bone scan be eliminated in patients with newly diagnosed prostate carcinoma in Japan who have serum PSA levels $<10 \mathrm{ng} / \mathrm{ml}$, and it is possible to omit baseline bone scans for patients with a Gleason grade $<2$ tumors or with a Gleason score $<6$. Their reports were very informative studies, however, PSA kits, the pathologist, the radiologist and the orthopedist were not so well standardized because of the multicenter study. Our study was done in a hospital, so we could analyze only 296 bone scan cases, but the levels of diagnosis were standardized. From the analysis of PSA, Gleason sum and clinical T stage, we concluded that we may not have to do bone scan for the patients whose PSA level is under $15 \mathrm{ng} / \mathrm{ml}$ (the possibility of bone metastasis is $10 \%$ from the simple linear regression analysis) or Gleason sum is under 5 .

Our results may also become useful to determine the indication for bone scan, and to explain the probability of bone metastasis to prostate cancer patients in Japan or other Asian countries. 


\section{References}

1. Middleton RG, Thompson IM, Austenfeld MS, Cooner WH, Correa RJ, Gibbons RP et al. Prostate cancer clinical guidelines panel summary report on the management of clinically localized prostate cancer. J Urol 1995; 154: 2144-8.

2. Baker LH, Hanks G, Gersherson D, Kantoff P, Lange P, Logothetis C et al. NCCN Prostate cancer guidelines. The National Comprehensive Cancer Network. Oncology (suppl) 1996; 10: 265-88.

3. Richie JP, Murphy GP, Walther P. Prostate cancer surgical practice guidelines. Society of Surgical Oncology practice guidelines. Oncology (Huntingt) 1997; 11:907-12.

4. O'Dowd GJ, Veltri RW, Orozco R, Miller MC, Oesterling JE. Update on the appropriate staging evaluation for newly diagnosed prostate cancer. J Urol 1997 158 (3 Pt 1):687-98.

5. CoffyDS. Similarities of prostate and breast cancer: Evolution, diet, and estrogens. Urology 2001; 57 (suppl) 1: 31-8.

6. Presti JR JC, Chang J, Bhargava V, Shinohara K. The optimal systematic prostate biopsy scheme should include 8 rather than 6 biopsies: results of a prospective clinical trial. J Uro 2000; 163:163-167.

7. Chybowski FM, Keller JJ, Bergstralh EJ, Oesterling JE. Predicting radionuclide bone scan findings in patients with newly diagnosed, untreated prostate cancer: 
prostate specific antigen is superior to all other clinical parameters. J Urol 1991; 145: $313-8$.

8. Pantelides ML, Bowman SP, George JR. Levels of prostate specific antigen that predict skeletal spread in prostate cancer. Br J Urol 1992; 70: 299-303.

9. Kosuda S, Yoshimura I, Aizawa T, Koizumi K, Akakura K, Kuyama J et al. Can initial prostate specific antigen determinations eliminate the need for bone scans in patients with newly diagnosed prostate carcinoma? Cancer 2002; 94: 964-72. 


\section{Legend for Figure}

Fig. 1

Probability of bone metastasis rate compared with PSA values 
Fig. 1

$\%$

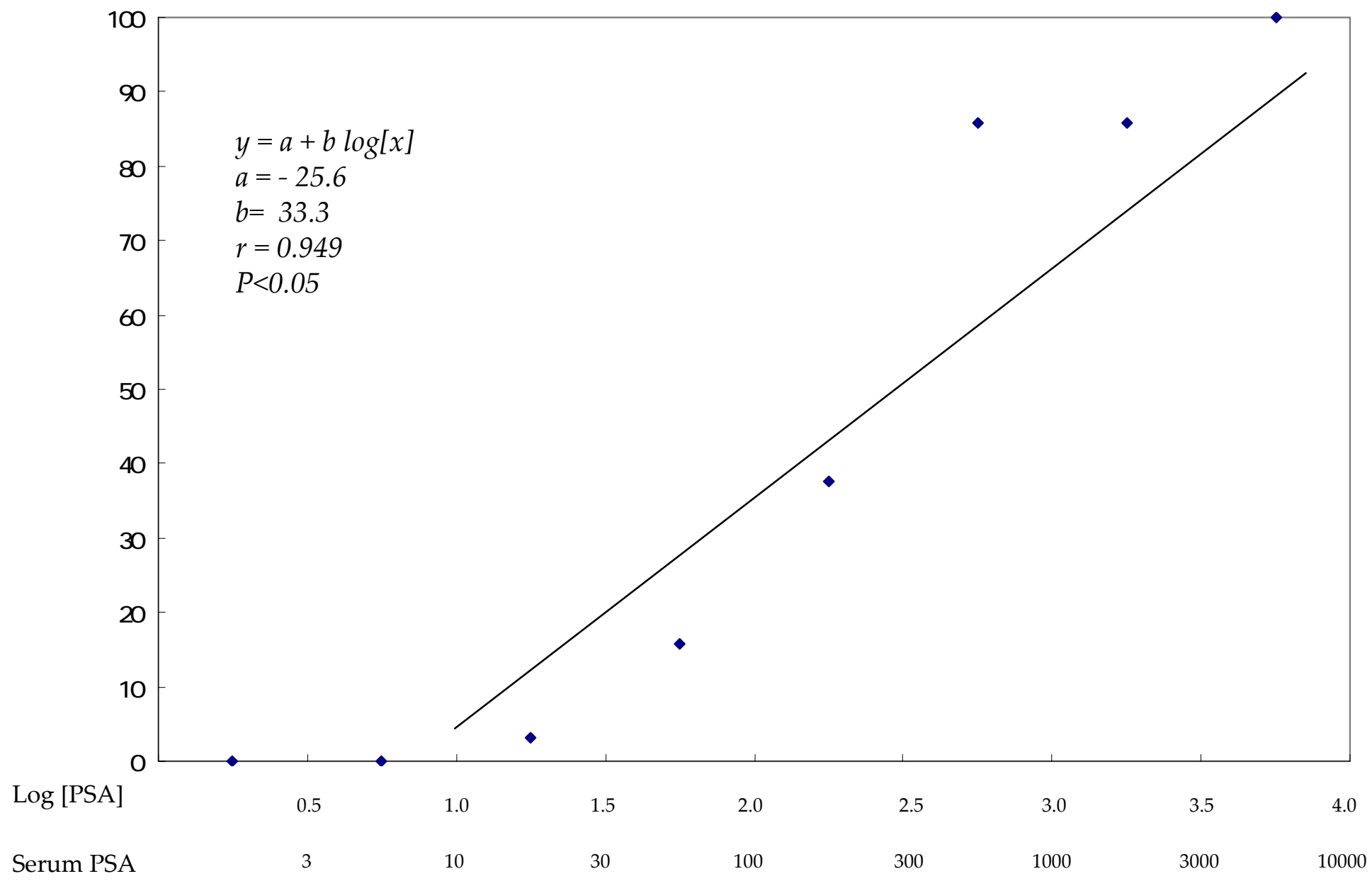


Age (mean, median), years, $\mathrm{n}=296$

PSA (mean, median), ng/ml, $\mathrm{n}=296$

Gleason sum (mean), $\mathrm{n}=238$

Clinical T stagen, $\mathrm{n}=296$

$\mathrm{T} 1$

$\mathrm{T} 2$

T3

$\mathrm{T} 4$

With bone metastasis

PSA level (mean, median), $\mathrm{n}=61$

Gleason sum (mean), $\mathrm{n}=48$

Without bone metastasis

PSA level (mean, median), $\mathrm{n}=235$

Gleason sum (mean), n=190
$72.5,72(45-90)$

$325.1,23.6(0.5-12288)$

$6.7(2-10)$

74

134

36

52

$61 / 296(20.6 \%)$

1365, 408 (16.6 - 12288)

$7.5(5-10)$

$235 / 296(79.4 \%)$

$55.1,16.8(0.5-2587)$

$6.5(2-10)$ 
Table 2

\begin{tabular}{ccccc} 
PSA $(\mathrm{ng} / \mathrm{ml})$ & $\log [\mathrm{PSA}]$ & Total number & Bone metastasis number & $\%$ \\
\hline $0-2.9$ & $0.0-0.49$ & 2 & 0 & 0 \\
$3.0-9.9$ & $0.5-0.99$ & 67 & 0 & 0 \\
$10.0-29.9$ & $1.00-1.49$ & 96 & 3 & 3.1 \\
$30.0-99.9$ & $1.50-1.99$ & 57 & 9 & 15.8 \\
$100-299$ & $2.00-2.49$ & 32 & 12 & 37.5 \\
$300-999$ & $2.50-2.99$ & 21 & 18 & 85.7 \\
$1000-2999$ & $3.00-3.49$ & 14 & 12 & 85.7 \\
$3000-9999$ & $3.50-3.99$ & 6 & 6 & 100 \\
$10000-$ & $4.00-$ & 1 & 1 & 100 \\
\hline & & 296 & 61 & 20.6
\end{tabular}


Table 3

\begin{tabular}{|c|c|c|c|c|c|c|c|c|c|}
\hline \multirow[b]{2}{*}{ PSA (ng/ml) } & \multirow[b]{2}{*}{$\log [\mathrm{PSA}]$} & \multicolumn{2}{|l|}{ GS 5} & \multicolumn{2}{|l|}{ GS 6} & \multicolumn{2}{|l|}{ GS 7} & \multicolumn{2}{|l|}{ GS $8<$} \\
\hline & & $\begin{array}{l}\text { Total } \\
\text { number }\end{array}$ & $\begin{array}{c}\text { Bone metastasis } \\
\text { (metastais rate \%) }\end{array}$ & $\begin{array}{c}\text { Total } \\
\text { number }\end{array}$ & $\begin{array}{l}\text { Bone metastasis } \\
\text { (metastais rate \%) }\end{array}$ & $\begin{array}{c}\text { Total } \\
\text { number }\end{array}$ & $\begin{array}{l}\text { Bone metastasis } \\
\text { (metastais rate \%) }\end{array}$ & $\begin{array}{c}\text { Total } \\
\text { number }\end{array}$ & $\begin{array}{l}\text { Bone metastasis } \\
\text { (metastais rate \%) }\end{array}$ \\
\hline $0-2.9$ & -0.49 & 1 & $0(0.0 \%)$ & 0 & $0(0.0 \%)$ & 1 & $0(0.0 \%)$ & 0 & $0(0.0 \%)$ \\
\hline $3.0-9.9$ & $0.50-0.99$ & 6 & $0(0.0 \%)$ & 29 & $0(0.0 \%)$ & 10 & $0(0.0 \%)$ & 4 & $0(0.0 \%)$ \\
\hline $10.0-29.9$ & $1.00-1.49$ & 15 & $0(0.0 \%)$ & 25 & $1(4.0 \%)$ & 16 & $0(0.0 \%)$ & 15 & $1(6.7 \%)$ \\
\hline $30.0-99.9$ & $1.50-1.99$ & 6 & $1(16.7 \%)$ & 6 & $1(16.7 \%)$ & 21 & $3(14.3 \%)$ & 14 & $3(21.4 \%)$ \\
\hline $100-299$ & $2.00-2.49$ & 3 & $1(33.3 \%)$ & 2 & $2(100 \%)$ & 8 & $3(37.5 \%)$ & 12 & $5(41.7 \%)$ \\
\hline $300-$ & $2.50-$ & 2 & $1(50.0 \%)$ & 2 & $2(100 \%)$ & 14 & $14(100 \%)$ & 13 & $10(76.9 \%)$ \\
\hline & & 33 & 3 & 64 & 6 & 70 & 20 & 58 & 19 \\
\hline
\end{tabular}

GS: Gleason sum 